\title{
Respiratory Distress Complicating Laryngeal Sarcoidosis
}

\author{
O. Benhoummad, O. Benlenda, M. EL Fakiri, and H. Nassik
}

\section{ABSTRACT}

Laryngeal involvement in sarcoidosis is rare but can be potentially dangerous. A Severe obstruction of the laryngeal tract may appear, sometimes requiring a tracheostomy. Systemic corticosteroid therapy is the treatment of choice for most patients. We report the case of a 25 -year-old patient with isolated laryngeal sarcoidosis for 12 years. She was admitted for respiratory distress. The nasofibroscopy revealed diffuse supraglottic edema, the medical care consisted of an intense surveillance and a corticosteroid therapy. Isolated laryngeal sarcoidosis is a condition that requires multidisciplinary consultation. It presents a challenge in terms of diagnosis, in terms of appropriate management of the respiratory tracts and in terms of treatment.

Keywords: Corticosteroid therapy, Laryngeal dyspnea, Laryngeal sarcoidosis, Morocco, Tracheotomy.

\section{INTRODUCTION}

Sarcoidosis is a multiorgan inflammatory granulomatous disease with predominantly mediastino-pulmonary expression. The damages of the oto-rhino-laryngeal sphere whose functional discomfort justifies its systematic research. The evolution is most often favorable with local corticosteroid therapy [1]. We report a case of isolated laryngeal sarcoidosis admitted in a respiratory distress which emphasis the diagnosis, the appropriate respiratory tracts management and the treatment.

\section{Clinical CASE}

This was a 25 -year-old woman with a history of anxiety disorder under treatment. She had been followed since 2008 for laryngeal sarcoidosis. The diagnosis was made on a staged biopsy of the larynx showing an appearance of gigantocellular granuloma without caseous necrosis. The patient was put on corticosteroid therapy for 2 years with clear clinical improvement, she stopped her treatment herself for 10 years. The patient was admitted to the ENT emergency room in July 2020 with a laryngeal dyspnea that had gradually set in for 3 weeks and worsened in the recumbency. It was associated with solids dysphagia without notion of dysphonia without other ENT signs. Everything evolved in a context of asthenia and low fever at $38^{\circ} \mathrm{C}$.

On general examination, the respiratory rate was 20 cycles per minute with slight intercostal and subclavian indrawing.
Submitted : October 13, 2020

Published : May 29, 2021

ISSN: $2593-8339$

DOI: $10.24018 /$ ejmed.2021.3.3.896

O. Benhoummad*

ENT department and head and neck surgery, CHU Agadir, Morocco.

(e-mail: o.benhoummad ${ }^{@}$ uiz.ac.ma)

O. Benlenda

Anesthesia and intensive care unit, CHU Agadir, Morocco.

M. EL Fakiri

ENT department and head and neck surgery, CHU Agadir, Morocco.

H. Nassik

Anesthesia and intensive care unit,

CHU Agadir, Morocco.

*Corresponding Author

The partial oxygen saturation was $94 \%$ in ambient air. The pleuropulmonary examination and the remainder of the physical examination were unremarkable.

The nasofibroscopy showed a hypertrophied and granite appearance of the laryngeal mucosa which predominated in the supraglottic level (epiglottis, aryepiglottic fold with the presence of a pedicled bud on the right arytenoid). The laryngeal pathway was reduced to more than $80 \%$ (Fig. 1).

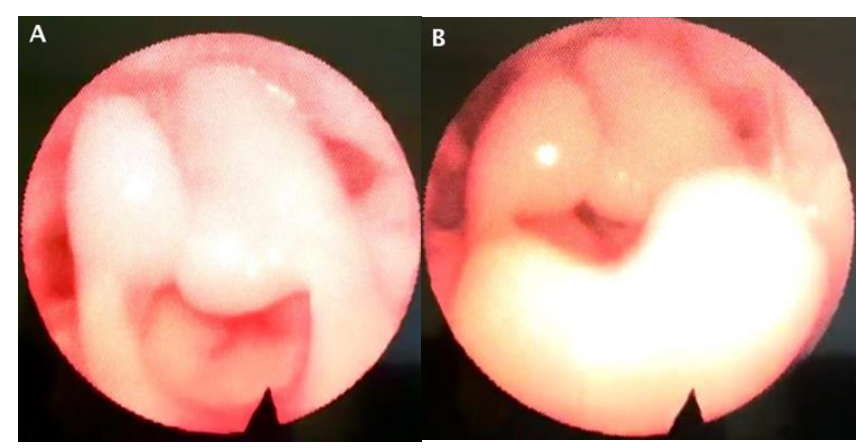

Fig. 1. A: Edematous whitish granite appearance of both arytenoids and aryepiglottic folds with a pedicled bud on the right arytenoid.

B: whitish edematous epiglottis.

Cervico-thoracic computed tomography revealed infiltration and mucous thickening of the epiglottis, aryepiglottic folds and supraglottic walls stenosing the laryngeal lumen with discreet mucous thickening of the nonstenosing vocal cords without associated pulmonary abnormalities (Fig. 2). The quantiferon assay was negative. 


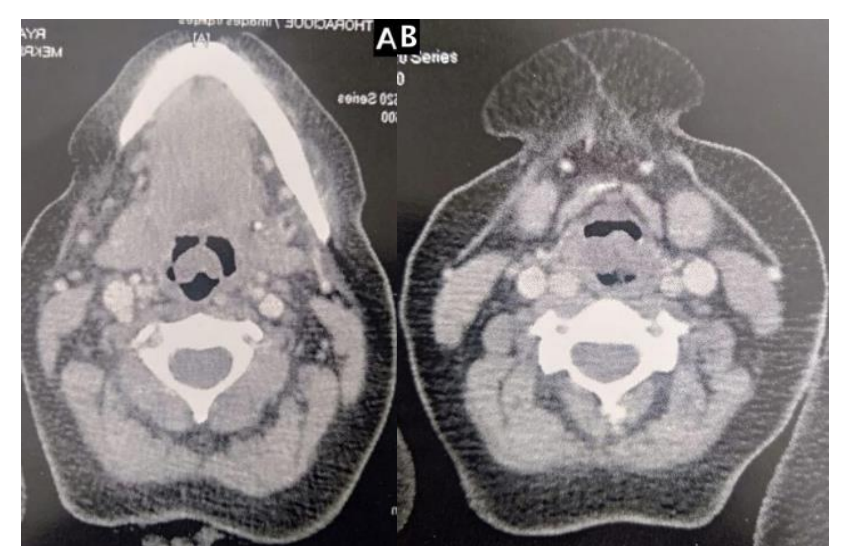

Fig. 2. A: epiglottis thickening up to $11 \mathrm{~mm}$ thick, B: narrowing of the lumen of the supraglottic laryngeal floor by thickening of the epiglottis, the aryepiglottic folds, and its posterior wall.

In view of this picture, the diagnosis retained was a exacerbation of isolated laryngeal sarcoidosis. The patient was hospitalized in the intensive care unit. She was put on oxygen therapy at $4 \mathrm{l} / \mathrm{min}$. Corticosteroid therapy with an intravenous bolus of methylprednisolone was initiated. It was replaced by oral corticosteroid therapy with prednisone at $1 \mathrm{mg} / \mathrm{kg}$ following bolus intolerance (occurrence of bradycardia). It was combined with treatment with Omeprazole at $40 \mathrm{mg} / \mathrm{day}$. Close clinical monitoring and a surgical tray for tracheostomy was prepared in the event of rapid decompensation. Respiratory improvement was noted after $72 \mathrm{~h}$ of treatment with $97 \%$ saturation in the open air. The patient was declared discharged with a corticosteroid treatment for 2 months then a degression spread over 3 months. A follow-up in ENT and internal medicine has been in progress for 3 months without aggravation.

\section{DISCUSSION}

Sarcoidosis, initially described in the 19th century, didn't change in its definition: systemic granulomatosis of unknown cause, characterized by the formation of immune granulomas in the affected organs, primarily the lung and lymphoid tissue. Sarcoidosis now appears as a multigene disease favored by environmental factors [2], [3]. Its onset is usually between 20 and 40 years old. It is slightly more common in women and people of African descent [4]. Its incidence varies by geographic region. The highest annual incidence of sarcoidosis has been observed in northern European countries (5 to 40 cases per 100,000 people) [5]. The entire mucosa of the upper respiratory tract may be involved: the nose, nasopharynx, oropharynx, and larynx [6]. Laryngeal involvement in sarcoidosis is rare, although potentially severe with a reported incidence of $0.6 \%$ to $1.3 \%$ [7]. It was in 1940 that Poe, for the first time, reported an observation of laryngeal sarcoidosis proven histologically [6]. Laryngeal sarcoidosis most often occurs during a multisystemic form of the disease, sometimes more than 10 years after the first manifestation. It can also be the inaugural manifestation of the disease as in our observation, or even be the only manifestation [6]. In the case of laryngeal involvement in the glottis, dysphonia is the most frequent initial symptom. Then come in the frequency order: dyspnea, related to an obstruction of the airway, dysphagia, probably by posterior invasion and attack of the pharynx, a non-productive cough, rarely stridor. Finally, there are a number of asymptomatic forms [6]. At the laryngeal level, sarcoidosis mainly affects the supraglottic region, especially the free edge of the epiglottis and the aryepiglottic folds, then the ventricular bands and the arytenoids, real damage to the vocal cord is rare [7]. However, cases of vocal cord paralysis secondary to peripheral neuropathy have been reported due to recurrent inferior laryngeal nerve damage [8]. The macroscopic appearance of the laryngeal involvement is considered pathognomonic: an enlarged, pale, pink and edematous epiglottis, often with a turban-like thickening [9]. The oriented biopsy, apart from any corticosteroid therapy, allows the diagnosis [10] while CT and MRI imaging of laryngeal sarcoidosis is not specific. It is mainly useful for the locoregional assessment of tumor or pseudotumor forms and for the diagnosis of associated sarcoidosis sites [10]. The diagnosis of laryngeal sarcoidosis is based on a set of clinical, biological and radiological arguments supported by histology which shows non-caseous granulomas after excluding other granulomatous diseases, in particular tuberculosis [2], [9]. The management of laryngeal sarcoidosis is difficult and unstandardized, primarily described in single case reports or very limited case series, and the results are difficult to assess due to the natural recurrent course of sarcoidosis. Laryngeal sarcoidosis treatment options can be divided into local or systemic treatments. The goal of treatment is preservation of the voice and prevention of respiratory tracts compromise [4]. The usual treatment is to take prednisone at doses of $1 \mathrm{mg} /$ $\mathrm{kg}$ body weight per day for 4 to 6 weeks, followed by a gradual decrease over 4 to 6 months. If the disease reappears, the cycle repeats [8]. The use of methylprednisolone as an intravenous bolus may be proposed in some particularly severe and urgent situations endangering a vital or sensory function (for example poorly tolerated laryngeal damage as in our case), or even in the event of a therapeutic impasse [3]. In practice, corticosteroid therapy is instaured according to the activity of the sarcoidosis, or the extent of the symptoms due to laryngeal damages [6]. Its treatment requires high doses of corticosteroids that last for several years. Nonresponders to steroids may benefit from experimental therapies: clofazamine, cytotoxic agents (methotrexate, azathioprine), intralesional steroid injection, radiotherapy. Surgery and endoscopic $\mathrm{CO}_{2}$ laser reduction can be used as a last resort [9]. Finally, tracheostomy may become necessary in the case of severe, recurrent and potentially fatal obstructions. Oral intubation can be an alternative, but it is not always possible as in our case, given the significant reduction of the essentially supraglottic laryngeal pathway [8], [9]. some therapeutic rules must be respected, the treatment is purely suspensive and a rebound when stopping the treatment is possible with all molecules [7]. In some cases, the disease progresses despite all attempts of treatment and it is therefore imperative to rule out another diagnosis or associated disease [7]. The evolution is unpredictable, ranging from stability or even spontaneous regression to recurrence or chronicity despite often long treatment [7], [10]. It can lead to atrophy of the laryngeal mucosa which may persist after disappearance of the granulomas [6] However, its evolution is far from being consistently favorable (complete remission is only observed in just over $50 \%$ of cases) [7]. The prognosis for ENT involvement in 
sarcoidosis is functional but laryngeal locations are lifethreatening, their long-term prognosis is often disappointing, and the patients are monitored over years [7], [10]. This case illustrates the potentially fatal nature of laryngeal sarcoidosis and the difficult decisions it presents to the various physicians involved in its management.

\section{CONCLUSION}

Isolated laryngeal sarcoidosis is described as involving not only the supraglottic regions but also the glottic and subglottic areas. The presentation, diagnosis and treatment options for laryngeal sarcoidosis are not standardized. In this case, systemic corticosteroid therapy was the treatment of choice with good outcome.

\section{DECLARATION OF INTEREST}

The authors declare that they have no conflicts of interest regarding this article.

\section{REFERENCES}

[1] M. Khedher, F. Said, A. Hamzaoui, M. Khanfir, M.H. Houman, «L'atteinte ORL au cours de la sarcoïdose», La Revue de médecine interne 39 (2018) A118-A252.

[2] P. Sèvea, M. Pavicc, «Sarcoïdose: quoi de neuf pour l'interniste en 2010?», La Revue de médecine interne 32 (2011) 71-72.

[3] C. Londnera, I. Zendaha,b, O. Freyneta, Z. Cartona, G. Diona, H. Nunesa, D. Valeyrea, «Traitement de la sarcoïdose», La Revue de médecine interne 32 (2011) 109-113.

[4] Y. Agrawal, DA. Godin, PC. Belafsky. «Cytotoxic Agents in the Treatment of Laryngeal Sarcoidosis: A Case Report and Review of the Literature», Journal of Voice (2006), Vol. 20, No. 3, pp. 481-484.

[5] E Piekarski, K Benali, F Rouzet. «Nuclear Imaging in Sarcoidosis», Semin Nucl Med (2018) 48:246-260.

[6] F. Visticot, L. Vanrenterghem, G. Montreuil, Py. Hatron, B. Devulder, «Sarcoïdose des voies ariennes supérieures, Revue de la littérature à propos de deux observations», La Revue Med Interne (1994) 15, 747 751.

[7] C. Chapelon Abric, " Localisations extrathoraciques graves de la sarcoïdose », La Revue de médecine interne 32 (2011) 80-85.

[8] ÁL Barreiro, JJ. Díaz Argüello, José A. F Martínez, A Martínez Ferreras. «Laryngeal Sarcoidosis: Unique Location or First Manifestation? », Acta Otorrinolaringol Esp. 2012;63(3):230-232.

[9] C. Davis, DV. Girzadas Jr, «Laryngeal sarcoidosis causing acute upper airway obstruction», American Journal of Emergency Medicine (2008) 26, 114.e1-114.e3.

[10] J.J. Braun, S. Riehm, A. Imperiale, A.-S. Schultz-Carpentier, A. Gentinea, F. de Blay. «Sarcoïdose des voies aérodigestives supérieure», Revue des Maladies Respiratoires (2011) 28, 164-173. 